\title{
Habilidades sociais de usuários de crack acolhidos em comunidades terapêuticas
}

\section{Adauto Motta Filho}

(D) https://orcid.org/0000-0002-3136-569x

Andresa Aparecida Francisquini de Sousa

(D) https://orcid.org/0000-0001-7577-3516

Randolfo dos Santos Junior ${ }^{2}$

(D) https://orcid.org/0000-0002-8029-0188

Júlio César André ${ }^{3}$

(D) https://orcid.org/0000-0002-0549-4527

Loiane Letícia dos Santos ${ }^{4}$

(DiD https://orcid.org/0000-0003-0571-0370

Instituição de Ensino Superior, Catanduva, SP, Brasil.

2 Faculdade de Medicina de São José do Rio Preto, Departamento de Psicologia, São José do Rio Preto, SP, Brasil.

${ }^{3}$ Faculdade de Medicina de São José do Rio Preto, Centro de Estudos e Desenvolvimento de Educação em Saúde, São José do Rio Preto, SP, Brasil.

${ }^{4}$ Instituto Municipal de Ensino Superior, Departamento de Psicologia, Catanduva, SP, Brasil.
Objetivo: buscou-se, por meio deste estudo, avaliar as habilidades sociais de usuários de crack em abstinência, vide a caracterização do perfil sociodemográfico e identificação de possíveis déficits nas habilidades sociais. Método: amostra de conveniência de 39 dependentes químicos, com idade média de 40,7 anos $( \pm 10,48)$ e acolhidos em comunidades terapêuticas no interior do estado de São Paulo. O estudo caracteriza-se como descritivo, transversal e com delineamento quantitativo. Os instrumentos utilizados foram Questionário Sociodemográfico e Padrão de Consumo; Escala de Apoio Social; Escala de Autoestima e Inventário de Habilidades Sociais (IHS). Resultados: este estudo apontou déficits em habilidades sociais no score geral (84,3\%) dos participantes e nos subcomponentes do inventário de habilidades sociais. Nas correlações de habilidades sociais e apoio social, primordialmente os fatores afetivos ( $p \leq 0,0156)$ e emocional ( $p \leq 0,0299$ ) apresentaram valor de significância estatística, ou seja, quanto maiores os índices de apoio social, maior os recursos em habilidades sociais. A idade avançada apresentou correlação negativa ( $p \leq 0,0167$ ) em habilidades sociais; quanto maior a idade, menor o repertório de HS. Conclusão: este estudo destaca a relevância do apoio social ao dependente químico como fator de melhor desempenho social, possibilitando subsídios para novas pesquisas na área.

Descritores: Crack; Comunidade Terapêutica; Habilidades Sociais; Saúde Mental.

\section{Como citar este artigo}

Motta Filho A, Sousa AAF, Santos Junior R, André JC, Santos LL. Social skills of crack users accepted in therapeutic communities. SMAD, Rev Eletrônica Saúde Mental Álcool Drog. 2020;16(2):1-11. doi:https://dx.doi.org/10.11606/issn.1806-6976.smad.2020.155394 


\title{
Social skills of crack users accepted in therapeutic communities
}

Objective: through this study, we aimed to assess the social skills of crack users in abstinence, by characterizing the demographic profile and identifying possible deficits in social skills. Method: with a convenience sample of 39 chemical dependents, with an average age of 40.7 years ( \pm 10.48), hosted in therapeutic communities in the countryside of the state of São Paulo. The study is characterized as descriptive, transversal, with a quantitative delineation. Sociodemographic Questionnaire and Consumption Standard; Social Support Scale; Self-esteem Scale and the Social Skills Inventory (SSI) were the instruments utilized. Results: this study pointed out deficits in social skills in the general score (84.3\%) of the participants and in the subcomponents of the social skills inventory. In the correlations of social skills and social support, affective $(p<0.0156)$ and emotional $(p<0.0299)$ factors were statistically significant, that is, the higher the social support indexes, the higher the social skills resources. The old age presented a negative correlation $(p<0,167)$ in social skills, that is, the older the age, the lower the social skills repertoire. Conclusion: this study highlights the relevance of social support to the chemical dependent as a factor of better social performance, allowing subsidies for new researches in the area.

Descriptors: Crack; Therapeutic Community; Social Skills; Mental Health.

\section{Habilidades sociales de usuarios de crack acogidos en comunidades terapéuticas}

\begin{abstract}
Objetivo: se buscó por medio de este estudio, evaluar las habilidades sociales de usuarios de crack en abstinencia, por medio, de la caracterización del perfil sociodemográfico e identificación de posibles déficits en las habilidades sociales. Método: con muestra de conveniencia de 39 dependientes químicos, con edad media de 40,7 años ( $\pm 10,48$ ), acogidos en comunidades terapéuticas en el interior del estado de São Paulo. El estudio se caracteriza como descriptivo, transversal, con delineamiento cuantitativo. Los instrumentos utilizados fueron Cuestionario Sociodemográfico y Estándar de Consumo; Escala de Apoyo Social; Escala de Autoestima y el Inventario de Habilidades Sociales - (IHS). Resultados: el presente estudio apunta a déficit en habilidades sociales en la puntuación general $(84,3 \%)$ de los participantes, y en los subcomponentes del inventario de habilidades sociales. En las correlaciones de habilidades sociales y apoyo social, primordialmente los factores afectivos $(p \leq 0,0156)$ y emocional $(p \leq 0,0299)$ presentaron valor de significancia estadística, o sea, cuanto mayores los índices de apoyo social, mayor los recursos en habilidades sociales. La edad avanzada presentó correlación negativa ( $p \leq 0,0167$ ) en habilidades sociales, o sea, cuanto mayor la edad, menor el repertorio de HS. Conclusión: este estudio destaca la relevancia del apoyo social al dependiente químico, como factor de mejor desempeño social, posibilitando subsidios para nuevas investigaciones en el área.
\end{abstract}

Descriptores: Crack; Comunidad Terapéutica; Habilidades Sociales; Salud Mental. 


\section{Introdução}

O transtorno por uso de substâncias psicoativas (SPAs) tem se mostrado uma condição multifacetada, com elementos cognitivos, comportamentais e fisiológicos de difícil controle tanto para o usuário como para a sociedade ${ }^{(1)}$. Especificamente em relação ao crack, é perceptível o elevado poder de compulsão e fissura quase incontrolável por sua rápida absorção, induzindo o usuário a sensações estimulantes e extremamente prazerosas com maior fugacidade(2).

O perfil mais comum entre usuários de crack são indivíduos do sexo masculino, jovens e adultos, com pouca escolaridade, desempregados, provenientes de famílias vulneráveis e de baixa renda. Diversos aspectos característicos são identificados nesse contexto de população usuária de crack, como aumento de agressividade, envolvimento em atividades ilegais, falta de comprometimento com responsabilidades básicas, degradação da higiene pessoal e, consequentemente, maiores indicadores de comorbidades psiquiátricas ${ }^{(3-6)}$.

Frente a esse contexto, o dependente da substância passa a sofrer discriminação e exclusão social crônicas causadas pelo estigma relacionado ao crack, fruto de uma representação social sem precedentes de mutação(7-8).

Segundo dados epidemiológicos de uma pesquisa nacional nas capitais brasileiras e no Distrito Federal em relação ao uso de crack, 370 mil brasileiros são usuários constantes, ou seja, fazem o uso uma vez por semana nas capitais brasileiras $(0,8 \%)$, sendo $14 \%$ crianças e adolescentes, aproximadamente 50 mil. Foi observado que $70 \%$ dos usuários compartilham ferramentas para o uso, como cachimbos e latas de refrigerante ou de cerveja. A região Nordeste apresentou maior prevalência do consumo de SPA, com cerca de 150 mil usuários da droga. A pesquisa ainda constatou que a contaminação pelo vírus HIV entre os usuários de crack no Brasil é oito vezes maior do que na população em geral( ${ }^{(9)}$.

Um estudo nacional sobre uso de substâncias mostrou que $0,7 \%$ da amostra (representando cerca de 800 mil pessoas) havia feito uso de crack nos últimos 12 meses e 1,3\% (representando cerca de 1,7 milhões de brasileiros) relatou uso de crack pelo uma vez em sua vida. Mas os dados podem ser ainda maiores, uma vez que esse estudo não envolveu participantes em situação de rua, uma parcela da população especialmente vulnerável ao uso da droga(10).

Com os crescentes índices epidemiológicos em relação ao crack, a Comunidade Terapêutica (CT) se tornou um local de procura por tratamento entre os usuários dessa substância. A CT é uma modalidade de atenção e cuidado integral ao dependente químico, com ambiente estruturado e sem a presença de substâncias ilícitas, ofertando a obtenção e o manejo da abstinência, com abordagem abrangente e agregando profissionais de diversas áreas do conhecimento cientifico(11).

O estigma ou estereótipo em relação aos usuários de crack é um dos fatores de resistência ao tratamento. Assim, ao vivenciar um sentimento disfórico e de baixa autoestima associado a um possível comportamento hostil dos profissionais quanto à sua doença e substância de escolha, tendem a ter dificuldade de adesão ao tratamento. A hostilidade dos profissionais pode estar associada à necessidade de rotular, à ausência de empatia ou, ainda, a uma visão conservadora e preconceituosa em relação ao dependente ${ }^{(7)}$.

Há evidências de que alguns elementos, como conflito interpessoal, expectativas, estados emocionais, frustrações, inibição social e pressão social, podem contribuir para dificultar a reinserção social do dependente químico. Elas podem consistir no déficit de Habilidades Sociais (HS) básicas para executar tarefas e atividades do cotidiano, dentre as mais simples e necessárias para a vida em comunidade(12). Déficits em HS repercutem em condições de sofrimento psicológico e comportamental, levando a prejuízos nas relações interpessoais ${ }^{(13)}$.

As habilidades sociais podem ser mencionadas como um conjunto de capacidades comportamentais aprendidas e que concretizam as interações sociais de forma assertiva. Com a aquisição de tais HS, o indivíduo passa a dominar um comportamento adequado e atencioso em relação às atitudes, comunicação, crenças, desejos e forma de expressar emoções e opiniões nas relações interpessoais, o que compõe todo o repertório de sociabilidade do indivíduo, que consequentemente se adapta aos padrões culturais e contextuais de suas vivências ${ }^{(14)}$.

A ausência deste componente de sociabilidade envolve tanto aspectos de verbalização quanto não verbais, correlacionando-se com a cognição, emoções e comportamentos mal adaptativos nas resoluções de problemas de interação social. Com a falta de treinamento e a não aquisição dessas habilidades, as recaídas podem ocorrer com maior frequência(15).

Desta forma, as habilidades sociais podem ser também exemplificadas a partir do paradigma de aprendizagem social, o qual preconiza que as habilidades podem ser desenvolvidas por meio de experiências interpessoais, da observação do desempenho de outros e a partir de um processo de assimilação mental de modelos de êxito. Essas experiências mediadas por processos cognitivos (crenças, percepções e pensamentos) influenciarão a obtenção de determinadas habilidades de forma particular e única para cada indivíduo(16).

Ser detentor de habilidades sociais, além de um fator de proteção e resiliência efetiva, possibilita 
interações com outras pessoas com efeitos positivos e satisfatórios(17). Nesse sentido, estudos indicam que comportamentos socialmente habilidosos podem resultar em menores detrimentos aos usuários, possibilitando maior interação, podendo contribuir com uma maior qualidade em suas relações sociais e, consequentemente, agregando mudanças significativas ao seu estilo de vida. Tais comportamentos adaptativos, sendo apreendidos e treinados, resultaram em melhor resposta no enfrentamento da doença(18-19).

A partir de então, uma taxonomia mais ampla e organizada em HS pode contribuir para melhores resoluções comportamentais: a) comunicação: elaborar e dar respostas a perguntas, retribuir e exaltar qualidades, pedir e dar devolutiva nas relações sociais, começar, manter e terminar uma conversação; b) civilidade: falar por favor, retribuir um gesto, apresentar-se, dar congratulação, retirar-se; c) assertivas de enfrentamento: relevar opinião, consentir com algo ou não, relevar e assumir erros, efetivar relação afetivo-sexual, terminar envolvimento amoroso, externar raiva e requerer modificação de comportamento, interação com figuras de superioridade, reflexão com críticas; d) empáticas: explanar apoio por meio de paráfrase, revelar sentimentos e dar apoio; e) de trabalho: coordenar grupos, comunicar-se em público, deliberar problemas, ter decisões e mediar conflitos; f) educativas e de expressão de sentimentos positivos: edificar amizade, manifestar amparo e trabalhar o amor(20).

Logo, dada a complexidade do tema, tem sido escopo em pesquisas, tanto no âmbito da atenção clínica como em estudos sobre drogas e contribuições para determinar dimensões entre os escores deficitários em HS e associações com a dependência de SPAs em adolescentes $^{(21-22)}$, alcoolistas ${ }^{(23-24)}$, mulheres(19,25), homens $^{(26)}$ e tabagistas(27-28), O resultado desses estudos tem salientado que a ausência em HS pode ser um fator notável ao uso de substâncias lícitas e ilícitas e que outras populações decorrentes de condições e contextos diversos devem ser pesquisadas(29).

Pela observação dos aspectos analisados, entende-se que avaliar e mensurar elementos cognitivos e HS mostra-se como uma necessidade tanto de autoconhecimento quanto a atentar-se a possíveis armadilhas mentais que possam vir a surgir(30). O investimento na comunicação e no diálogo produz aumento nas relações interpessoais e no autocuidado intrapessoal, possibilitando aos dependentes de SPAs a recuperação da autoestima e autoeficácia, fatores essenciais de proteção e prevenção a possíveis recaídas, facultando a reinserção ao convívio social saudável(31).

Portanto, dada a importância em estudar novos métodos de tratamento e possibilidades precursoras para o cuidado integral aos indivíduos usuários de crack, este estudo visa realizar um levantamento dos dados sociodemográficos de pacientes em abstinência, identificar possíveis déficits em habilidades sociais e, dessa forma, correlacionar as variáveis apoio social e autoestima para compreender quais aspectos servem como proteção e quais promovem vulnerabilidade ao uso e, consequentemente, a recaídas.

\section{Método}

O estudo caracteriza-se como descritivo, transversal e com delineamento quantitativo. Após a aprovação do comitê de ética (Parecer no 2.900.192), foram realizadas coletas de dados em duas comunidades terapêuticas no interior do estado São Paulo.

A amostra de conveniência foi composta por 46 dependentes químicos, contudo foram excluídos do estudo sete participantes, devido ao tempo de abstinência e por não terem feito o uso de crack, em consonância com os critérios de exclusão e inclusão, totalizando 39 participantes.

Os critérios de inclusão foram dependentes químicos, usuários de crack, maiores de 18 anos e do sexo masculino. E os critérios de exclusão foram participantes acolhidos das instituições que não apresentassem, no mínimo, 10 dias de abstinência. Os instrumentos utilizados foram:

1. Questionário de dados sociodemográficos e padrão de consumo. Este instrumento, de autoria dos pesquisadores, foi construído com o objetivo de caracterizar os participantes: idade, estado civil, grau de escolaridade, religião, tempo de abstinência, idade quando do primeiro uso, frequência deste, utilização de substâncias com familiares, pensamentos suicidas, problemas com a justiça, entre outros itens.

2. Escala de Apoio Social. Determina indicadores multidimensionais nos laços sociais, trazendo aspectos funcionais das relações sociais. Composto por 19 itens que compreendem cinco dimensões funcionais de apoio social: material, afetiva, emocional, interação social positiva e informação. Esse instrumento abrange apoio social: material (quatro perguntas) - provisão de recursos práticos e ajuda material; afetiva (três perguntas) - demonstrações físicas de amor e afeto; interação social positiva (quatro perguntas) - contar com pessoas com quem relaxar e divertir-se; emocional (quatro perguntas) - habilidade de rede social em satisfazer as necessidades individuais em relação a problemas emocionais, por exemplo situações que exijam sigilo e encorajamento em momentos difíceis da vida; informação (quatro perguntas) - contar com pessoas que 
aconselhem, informem e orientem. Para todas as perguntas, cinco opções de resposta foram apresentadas: 1 "nunca"; 2 "raramente"; 3 "às vezes"; 4 "quase sempre" e 5 "sempre"(32).

3. Escala de Autoestima. A dimensão de autoestima vem sendo amplamente realizada por meio da Escala de Autoestima de Rosemberg (1965), conceitualizada como um instrumento que possibilita medir e classificar o nível de autoestima em baixo, médio e alto. A escala é composta por 10 sentenças fechadas, sendo cinco referentes à "autoimagem" ou "autovalor" positivos e cinco referentes à "autoimagem negativa" ou "autodepreciação". As sentenças são dispostas no formato Likert de quatro pontos, variando entre "discordo totalmente" ou "discordo" e "concordo totalmente" ou "concordo". Com relação à pontuação, quanto maior o escore obtido na escala, maior o nível de autoestima do indivíduo(33-34).

4. Inventário de Habilidades Sociais. É um instrumento de autorrelato, o qual avalia dimensões situacionais e comportamentais e que irá identificar as classes e subclasses de habilidades sociais que se encontram como deficitárias ou como elemento disponível no repertório do indivíduo. Composto de 38 itens, cada uma descreve uma situação de relação interpessoal e uma demanda de habilidade para reagir àquela situação. O respondente deve estimar a frequência com que reage da forma sugerida em cada item, considerando o total de vezes que se encontrou na situação descrita, e estimar a frequência de sua resposta em uma escala Likert com cinco pontos, variando de zero (nunca ou raramente) a quatro (sempre ou quase sempre). Em alguns itens há um fraseado "negativo", ou seja, uma pontuação mais alta indica déficit de habilidades sociais, devendo-se, nestes, inverter a pontuação para a obtenção do zero. $O$ instrumental produz um escore geral referenciado em termos de percentis e escores em cinco subescalas de habilidades sociais( ${ }^{(35)}$.

Este estudo foi realizado em duas etapas principais, devido à coleta de dados ser realizada em locais e cidades distintas.

10 Etapa: Apresentação em grupo, ou seja, com todos os acolhidos das instituições. Informou-se do que se trata o estudo nas duas comunidades terapêuticas, acompanhado por um membro da equipe de ambas as instituições.

20 Etapa: Após a orientação individual sobre os objetivos da pesquisa e informações éticas, dada a concordância com a assinatura do Termo de
Consentimento Livre Esclarecido, iniciou-se a coleta de amostras, que se configurou individualmente por meio da aplicação de um roteiro de entrevista semiestruturado com os instrumentos descritos e uma média de tempo de 40 a 50 minutos cada aplicação.

Para a análise dos dados sociodemográficos foi utilizada a estatística descritiva e para correlação foi realizado o teste de Spearman, com intervalo de confiança de 95\% (IC95\%), considerando significativo valor $p \leq 0,05$. As análises foram realizadas utilizando o software GraphPad Prism versão 6.

\section{Resultados}

A amostra total deste estudo foi composta por 39 participantes, com idade média de 40,7 anos $( \pm 10,48)$, sendo a idade mínima 18 anos e a máxima 64 anos. A amostra composta unicamente pelo sexo masculino caracteriza-se primordialmente por participantes brancos (56,5\%), católicos (59\%) e com escolaridade de primeiro grau (48,7\%). Quanto ao estado civil $(51,2 \%)$ são solteiros e a atividade profissional predominante foi serviços gerais $(20,5 \%)$.

Tabela 1 - Descrição em frequência ( $n$ ) e percentual (\%) dos dados sociodemográficos da amostra de participantes usuários de substâncias psicoativas das comunidades terapêuticas. Catanduva e Novo Horizonte, SP, Brasil, 2018

\begin{tabular}{|c|c|c|}
\hline Variáveis & $\mathbf{n}$ & $(\%)$ \\
\hline \multicolumn{3}{|l|}{ Religião } \\
\hline Agnóstico & 09 & 23 \\
\hline Católico & 23 & 59 \\
\hline Evangélico & 07 & 18 \\
\hline \multicolumn{3}{|l|}{ Escolaridade } \\
\hline $1^{\circ} \mathrm{Grau}$ & 19 & 48,7 \\
\hline $2^{\circ} \mathrm{Grau}$ & 18 & 46,1 \\
\hline Ensino Superior Incompleto & 01 & 2,6 \\
\hline Sem escolaridade & 01 & 2,6 \\
\hline \multicolumn{3}{|l|}{ Estado Civil } \\
\hline Com companheiro & 10 & 25,7 \\
\hline Divorciado & 07 & 18 \\
\hline Viúvo & 02 & 5,1 \\
\hline Solteiro & 20 & 51,2 \\
\hline \multicolumn{3}{|l|}{ Cor } \\
\hline Branco & 22 & 56,5 \\
\hline Preto & 06 & 15,4 \\
\hline Pardo & 11 & 28,2 \\
\hline \multicolumn{3}{|l|}{ Atividade Profissional } \\
\hline Serviços Gerais & 08 & 20,5 \\
\hline Trabalhador Rural & 06 & 15,4 \\
\hline Vendedor & 02 & 5,1 \\
\hline Operador industrial & 05 & 12,9 \\
\hline Operador de máquinas & 02 & 5,1 \\
\hline Auxiliar de produção & 05 & 12,9 \\
\hline Autônomo & 09 & 23 \\
\hline Solador & 02 & 5,1 \\
\hline
\end{tabular}


A Tabela 2 apresenta as características e histórico do abuso de substância dos participantes da amostra (com idade média de 53,85 anos), no qual a idade de início do uso da substância foi de 13 a 18 anos, 77\% apontaram ter histórico do uso familiar, o uso acompanhado de um familiar foi representado por $74,3 \%$ e o uso de SPAs com mais de um familiar por $43,5 \%$. A variável internações anteriores traz que $69,2 \%$ já utilizaram esse recurso de tratamento e $84,6 \%$ têm algum tipo de apoio familiar, enquanto 53,9\% contam com apoio de amigos.

Tabela 2 - Descrição em frequência (n) e percentual (\%) das características e histórico do abuso de substância dos integrantes das comunidades terapêuticas. Catanduva e Novo Horizonte, SP, Brasil, 2018

\begin{tabular}{|c|c|c|}
\hline Variáveis & $\mathbf{N}$ & $(\%)$ \\
\hline \multicolumn{3}{|l|}{ Idade de início do uso da substância } \\
\hline Terceira Infância (8 a 13 anos) & 07 & 18 \\
\hline 13 anos a 18 anos & 21 & 53,8 \\
\hline$>18$ anos & 11 & 28,2 \\
\hline \multicolumn{3}{|l|}{ Situação de Rua } \\
\hline Sim & 18 & 46,1 \\
\hline Não & 21 & 53,9 \\
\hline \multicolumn{3}{|l|}{ Internações Anteriores } \\
\hline Sim & 27 & 69,2 \\
\hline Não & 12 & 30,8 \\
\hline \multicolumn{3}{|l|}{ Histórico de uso familiar } \\
\hline Sim & 30 & 77 \\
\hline Não & 09 & 23 \\
\hline \multicolumn{3}{|c|}{ Histórico de uso familiar (Grau de Parentesco) } \\
\hline Pai & 02 & 5,2 \\
\hline Mãe & 02 & 5,2 \\
\hline Irmãos & 06 & 15,3 \\
\hline Mais de um familiar & 17 & 43,5 \\
\hline Outros (tios, primos) & 12 & 30,7 \\
\hline \multicolumn{3}{|l|}{ Histórico de uso com familiar } \\
\hline Sim & 29 & 74,3 \\
\hline Não & 10 & 25,7 \\
\hline \multicolumn{3}{|l|}{ Pensamentos suicidas } \\
\hline Sim & 17 & 43,6 \\
\hline Não & 22 & 56,4 \\
\hline \multicolumn{3}{|l|}{ Apoio de Familiares } \\
\hline Sim (mais de um familiar) & 33 & 84,6 \\
\hline Não & 05 & 12,9 \\
\hline Não citou & 01 & 2,5 \\
\hline \multicolumn{3}{|l|}{ Apoio de Amigos } \\
\hline Sim (mais de um amigo) & 21 & 53,9 \\
\hline Não & 18 & 46,1 \\
\hline
\end{tabular}

A Tabela 3 apresenta os resultados dos instrumentos utilizados por esse estudo e, como pode ser observado na Escala de Autoestima de Rosemberg, os scores gerais (17,1\%). Já na Escala de Apoio Social o escore geral apresentado foi de $14,1 \%$. O instrumento de autorrelato Inventário de Habilidades Sociais indicou de modo geral escore deficitário $(84,3 \%)$.

Tabela 3 - Apresentação de indicadores de pontuações mínimas, máximos, mediana e média das Escalas de Autoestima Rosemberg, Apoio Social e Inventário de Habilidades Sociais, resultados apresentados pelos usuários de substâncias psicoativas das comunidades terapêuticas. Catanduva e Novo Horizonte, SP, Brasil, 2018

\begin{tabular}{lccc}
\hline & Min - Max & Mediana & Média $( \pm)^{*}$ \\
\hline $\begin{array}{l}\text { Escala de Autoestima } \\
\text { Rosemberg }\end{array}$ & & & \\
$\quad$ Score Total & $11-25$ & 17 & $17,1( \pm 2,7)$ \\
Escala de Apoio Social & & & \\
$\quad$ Score Total & $5,8-19$ & 14,8 & $14,1( \pm 3,6)$ \\
Inventário de Habilidades & & & \\
Sociais & & & \\
$\quad$ Score Total & $43-119$ & 81 & $84,3( \pm 18,3)$ \\
\hline *Desvio Padrão & & &
\end{tabular}

A Tabela 4 demonstra a Descrição em frequência ( $n$ ) e percentual (\%) dos dados de cada fator do Inventário de Habilidades Sociais (IHS). Os componentes indicaram repertório bem elaborado (18\%), bom repertório acima da mediana (12,9\%), bom repertório abaixo da mediana $(7,6 \%)$ e déficit em habilidades sociais $(61,5 \%)$.

Tabela 4 - Descrição em frequência (n) e percentual (\%) dos dados de cada fator do Inventário de Habilidades Sociais (IHS) apresentados pelos usuários de substâncias psicoativas das comunidades terapêuticas. Catanduva e Novo Horizonte, SP, Brasil, 2018

\begin{tabular}{lcc}
\hline \multicolumn{1}{c}{ Percentil Inventário de Habilidades Sociais } & N & (\%) \\
\hline Repertório bem elaborado (100 a 75) & 07 & 18 \\
Bom repertório acima da mediana (75 a 50) & 05 & 12,9 \\
Bom repertório abaixo da mediana (50 a 25) & 03 & 7,6 \\
Déficit em Habilidades Sociais & 24 & 61,5 \\
\hline
\end{tabular}

A Tabela 5 apresenta a correlação entre indicadores de Habilidades Sociais e os fatores da Escala de Apoio Social, Autoestima, idade início do uso da substância e idade atual dos participantes da amostra. Evidenciaram-se aspectos relevantes nas variáveis Escala de Apoio Social nos fatores afetivo ( $p \leq 0,0156)$ e emocional ( $p \leq 0,0299)$ e a variável idade atual ( $p \leq 0,0167$ ).

É importante salientar que os resultados deste estudo demostraram associações diretas nas dimensões de habilidades sociais e apoio social, primordialmente os fatores afetivos ( $p \leq 0,0156$ ) e emocional ( $p \leq 0,0299$ ) tendo valor de significância estatística. Esses resultados apontam que quanto maiores os índices de apoio social, maiores são as habilidades sociais. Para estes acolhidos nas comunidades terapêuticas, ter apoio social é positivo 
e de suma importância para o enfrentamento da doença e desempenho de competência social.

Tabela 5 - Correlação entre indicadores de Habilidades Sociais e os fatores da Escala de Apoio Social, Autoestima, idade início do uso da substância e idade atual, indicadores de intervalo de confiança inferior e superior dos usuários de substâncias psicoativas das comunidades terapêuticas. Catanduva e Novo Horizonte, SP, Brasil, 2018

\begin{tabular}{lcccc}
\hline \multicolumn{1}{c}{ Variáveis } & $\begin{array}{c}\text { Habilidades } \\
\text { Sociais }\end{array}$ & $\mathbf{p}$ & $\begin{array}{c}\text { Limite } \\
\text { Superior }\end{array}$ & $\begin{array}{c}\text { Limite } \\
\text { Inferior }\end{array}$ \\
\hline Escala de Apoio Social & & & & \\
Fator Afetivo & 0,3848 & $0,0156^{*}$ & 0,0691 & 0,6304 \\
Fator Emocional & 0,3481 & $0,0299^{*}$ & 0,0269 & 0,6042 \\
Fator Informação & 0,0131 & 0,9370 & $-0,3125$ & 0,3359 \\
Fator Integração Social & 0,0536 & 0,7460 & $-0,2755$ & 0,3714 \\
Fator Material & 0,0835 & 0,6133 & $-0,2475$ & 0,3970 \\
Escala de Autoestima & & & & \\
Score Total & 0,1396 & 0,2380 & $-0,1396$ & 0,4871 \\
Idade de Início do uso & $-0,1531$ & 0,3522 & $-0,4548$ & 0,1801 \\
Idade Atual & $-0,3810$ & $0,0167^{*}$ & $-0,6277$ & 0,0647 \\
\hline *p <0,05 & & & &
\end{tabular}

\section{Discussão}

O uso de substância psicoativa na adolescência tem aumentado a vulnerabilidade e dependência, haja vista os reflexos da evasão escolar(36). Outro estudo, com 35 poliusuários em tratamento ambulatorial, avaliou as HS e possíveis correlações com o uso de SPAs destes, apresentando relações significativas nos fatores relacionados a habilidades de enfrentamento e autoafirmação com risco e dificuldades em conversação e desenvoltura social no Inventário de Habilidades Sociais (IHS), apontando ausência de HS para lidar com situações que demandam agilidade social(37).

Conota-se, por meio das variáveis histórico de uso familiar, grau de parentesco e histórico de uso com familiar, o poder de influenciar no processo de abuso de SPAs de forma grave, pois a construção da identidade do adolescente no contexto familiar é amplamente significativa e de suma importância. Desta forma, tem-se verificado que essas famílias que apresentam histórico de uso de SPAs e, consequentemente, a ausência de valores saudáveis influenciam a formação desse indivíduo. Ainda mais por ser um período de transformação biopsicossocial de constituição da personalidade, o uso de drogas pode fomentar prejuízos significativos no desenvolvimento desse sujeito(38).

O processo de desenvolvimento do indivíduo se dá no ambiente familiar primário, haja vista que as respostas sociais são assimiladas pelo traslado de comportamentos observados e vivenciados juntamente com outras pessoas do meio familiar. Nesse sentido, uma família com histórico de uso de drogas apresenta o processo de aprendizado e aquisições de estratégias em habilidades sociais altamente deficitários ${ }^{(39)}$.

Outro estudo de Schneider e Andretta(40) trouxe correlação de aspectos associados a déficits em HS, autocontrole, agressividade a situações aversivas e uso abusivo de drogas. Os resultados apontam que os indivíduos que tinham pai, irmão ou outros familiares que mantiveram relações de histórico de uso de drogas apresentavam maior risco para o início e a propagação de seu consumo abusivo. Por meio das relações familiares altamente conflituosas, esse indivíduo aprende a se relacionar de forma não assertiva, encontrando dificuldades em expor e controlar seus comportamentos.

$\mathrm{O}$ aspecto multifacetado da autoestima, por sua vez, indicou níveis baixos gerais dos participantes, sugerindo indícios de que possíveis alterações estejam associadas ao uso contínuo de SPAs e local de tratamento. Perfazendo a análise fatorial que a escala propôs, confirma-se o que está sendo observado nessa população usuária de crack de baixa autoestima devido à autodestruição e perturbação causadas pelo vasto impacto pernicioso da substância no usuário(33).

Abrangendo o aspecto de apoio social dos participantes do estudo, apontou-se que em algum momento da vida eles podem contar com o apoio de familiares e amigos para situações estressoras. Verificou-se, nos subcomponentes da escala, evidências que os laços sociais têm influência significativa na manutenção e ascensão da qualidade de vida desses usuários e que a ausência desse recurso no pós-alta pode ser determinante para lapsos e recaídas(41).

Verificou-se a incidência deficitária em habilidades sociais nos usuários de crack em abstinência, coincidindo com outras pesquisas que constatam, nesta população, déficits em HS mais significativos devido também aos danos nocivos causados pela substância. Estudos comparativos com dependentes e não dependentes identificaram resultados de scores mais baixos nessa população de dependente. O registro de déficit em habilidades sociais tem sido confirmado em associação a diferentes condições ${ }^{(42)}$.

Em Portugal, um estudo verificou condutas antissociais e delitivas e habilidades sociais em contextos diferentes, divididas em três subgrupos: não usuários de substâncias ilícitas, dependentes em comunidade terapêutica e em casa de acolhimento para redução de danos. Verificou-se a existência de diferenças deficitárias quanto à amostra de dependentes no nível das habilidades sociais nos escores fatoriais enfrentamento e autoafirmação com risco, um valor baixo obtido pelo subgrupo dos dependentes químicos em CT e níveis 
médios inferiores de conversação e desenvolvimento social(43) no grupo da casa de acolhimento.

Outro estudo realizou uma revisão sistemática nas bases Medline Complete, Scopus, IBECS, Index Psi e LILACS, resultando em 89 artigos, dos quais 13 foram analisados na íntegra, sendo selecionados 5 artigos. Conclui-se, nesta revisão, que usuários de drogas apresentam escores negativos em HS e que o treinamento em HS pode ter subterfúgios eficazes, denotando a evolução do cuidado que vem sendo empregado ao tratamento de tal doença(44).

Dada a crescente em estudos nacionais abordando a temática apoio social, nota-se o esboço que é empregado para verificar a ajuda a esses indivíduos. Esse apoio percebido faz menção à percepção de auxílio que se tem em determinada situação difícil e estressora enfrentada. Portanto, o apoio social provavelmente estabelece aspectos singulares da personalidade, levando em conta que os eventos que ocorrem na vida podem comprometer as habilidades e que esse apoio seja efetivo(45).

Além disso, a somatória de complicações decorrentes da fusão dos parâmetros de uso compulsivo que o crack proporciona na vida desses usuários é incontestável. É ampla a gama de danos, principalmente no que tange intrinsicamente à ruptura do vínculo familiar, levando à extrema fragilidade no suporte social(46).

A variável idade atual apresentou correlação negativa, ou seja, quanto maior a idade menores os indicadores de habilidades sociais. Ao avaliar a população de idade avançada com déficits em habilidades sociais, denotou-se a escassez na literatura e verificou-se que a presente amostra nas comunidades terapêuticas decorrentes do envolvimento com uso de crack trazem prejuízos aos usuários, indicando ausência de habilidades sociais, se comparado com os mais jovens, limitando, desta forma, o desempenho social com desenvoltura. Diversos fatores, como início de uso precoce, herança genética e institucionalização, podem contribuir negativamente para segregar esse repertório de sociabilidade ${ }^{(47)}$.

Estudos conotam a importância das mudanças de comportamentos em pessoas com tal faixa etária, sendo imprescindível essa integração social para a melhora de saúde física e mental desses usuários ${ }^{(48)}$.

Em virtude da relevância em se buscar possibilidades inovadoras de tratamento mais eficazes com usuários de substâncias, esse tema vem sendo alvo de muitos pesquisadores na última década. Recentemente, um estudo revelou circunstâncias associadas a escores deficitários no Inventário de Habilidades Sociais (IHS) entre usuários de crack. Com amostragem de 519 indivíduos, sendo 497 (95,8\%) homens e 22 (4,2\%) mulheres. Constatou-se que 52,8\% apresentaram déficit. Outro aspecto importante constatado foram as relações deficitárias em HS com luto da perda de filhos e envolvimento em atividades ilegais. Os escores trazem que o elevado apoio social está relacionado às habilidades sociais, dada consonância com este estudo(49).

Portanto, faz-se perceptível as muitas discussões envoltas ao tema acerca de fatores de risco e de proteção de dependentes químicos e as HS estão vinculadas a ambos os aspectos. Um estudo apontou a relação das HS divididas em dois grupos, usuários de crack e não usuários. No grupo de usuários, os resultados identificaram maiores habilidades sobre enfrentamento com risco e menores habilidades de conversação e desenvoltura social. Além do mais, o estudo apontou que ter filhos e não ter um companheiro (solteiro ou divorciado) também aumentou a probabilidade do uso do crack $^{(50)}$.

Por fim, é importante considerar as limitações deste estudo, que correspondem à restrição dos locais da pesquisa, tornando-se evidente que outros estudos empíricos sejam realizados abrangendo outras comunidades e gênero; de modo que se tenha uma compreensão ampliada do fenômeno, garantindo a efetividade de programas cientificamente embasados na prevenção e cuidado integral ao tratamento de dependentes químicos.

\section{Conclusão}

Esta pesquisa possibilitou, por meio dos resultados encontrados, a compreensão de diversos aspectos característicos dessa população que podem estar relacionados ao desencadeamento do repertório do uso de crack. Verificou-se que, quanto maior apoio social melhor o repertório de habilidades sociais, possivelmente influenciados pela necessidade de reestabelecer os vínculos no auxílio ao enfrentamento da abstinência.

Identificou, ainda, que pessoas com idade avançada possuem menores recursos em HS do que os participantes mais jovens. Averígua-se no estudo que alguns elementos como idade precoce no início do uso de substâncias psicoativas, ter histórico do uso familiar, o uso acompanhado de um familiar, baixa autoestima, falta de apoio social e déficits de recursos em HS apresentaram relevância e estão relacionados a múltiplos fatores de risco e vulnerabilidades para possíveis recaídas.

Desta forma, este trabalho buscou contribuir na compreensão da dependência química e associações com as HS em indivíduos na comunidade terapêutica, obtendo como desdobramentos a necessidade da inclusão da avaliação e treinamento em HS no método de intervenção terapêutica desses locais de tratamento, no intuito de aumentar os repertórios de enfrentamento 
ao uso da droga e diminuir as recaídas por meio da reinserção ao convívio social.

\section{Referências}

1. American Psychiatric Association. Manual diagnóstico e Estatístico de Transtornos Mentais: DSM-5. 5ed. Porto Alegre: Artmed; 2014. p. 483-5.

2. Cordeiro DC, Laranjeira R, Figle NB. Crack. In: Figlie NB, Bordin S, Laranjeira R, organizadores. Aconselhamento em Dependência Química. São Paulo: Roca; 2015. p. 65-8.

3. Fundação Oswaldo Cruz (BR). Crack: pesquisa identifica perfil, comportamento e vulnerabilidade de usuários. [Internet ]. 2016. [Acesso 11 ago 2018]. Disponível em: https://portal.fiocruz.br/noticia/ crack-pesquisa-identifica-perfil-comportamento-evulnerabilidade-de-usuarios.

4. Guimarães CF, Santos DVV dos, Freitas RC de, Araújo RB. Perfil do usuário de crack e fatores relacionados à criminalidade em unidade de internação para desintoxicação no Hospital Psiquiátrico São Pedro de Porto Alegre (RS). Rev Psiquiatr Rio Gd Sul. [Internet]. 2008 ago. [Acesso 12 ago 2018]; 30(2): 101-8. Disponível em: http://dx.doi.org/10.1590/S0101-81082008000300005. 5. Oliveira LG, Nappo SA. Caracterização da cultura de crack na cidade de São Paulo: padrão de uso controlado. Rev Saúde Pública. [Internet]. 2008 ago. [Acesso 12 ago 2018]; 42(4): 664-71. Disponível em: http://dx.doi. org/10.1590/S0034-89102008005000039.

6. Zanotto DF, Assis FB. Perfil dos usuários de crack na mídia brasileira: análise de um jornal e duas revistas de edição nacional. Physis. 2017 jul. [Acesso 11 ago 2012]; 27(3): 771-92. Disponível em: http://dx.doi. org/10.1590/s0103-73312017000300020.

7. Ronzani TM, Noto AR, Silveira PS da. Reduzindo o estigma entre usuários de drogas: guia para profissionais e gestores. [Internet ]. 2014. [Acesso 15 maio 2018]; 23 p. Disponível em: http://www.ufjf.br/ crepeia/files/2014/05/MIOLO_Reduzindo-o-Estigma_ ED-ATUALIZADA-baixa.pdf

8. Ministério da Saúde. Secretaria Nacional de Polícias Sobre Drogas (BR). O uso de substâncias psicoativas no Brasil: Modulo 1. 13. ed. Brasília: MJC; 2018. 146 p.

9. Fundação Oswaldo Cruz (BR). Estimativa do número de usuários de crack e/ou similares nas capitais do país. [Internet]. 2013. [Acesso 20 maio 2018]. Disponível em: https://portal.fiocruz.br/noticia/maior-pesquisa-sobre-crackja-feita-no-mundo-mostra-o-perfil-do-consumo-no-brasil 10. Instituto Nacional de Ciência e Tecnologia Para Políticas Públicas de Álcool e Outras Drogas (BR). II Levantamento Nacional de Álcool e Drogas [Internet]. 2012. São Paulo: Unifesp; 2014. 85 p. [Acesso 23 maio 2018]. Disponível em: <https://inpad.org.br/wp-content/uploads/2014/03/ Lenad-II-Relatório.pdf>.
11. Ministério da Saúde (BR). Secretaria Nacional de Polícias Sobre Drogas. Modalidades de Tratamento e Encaminhamento: modulo 6. 13. ed. Brasília: MJC; 2018. 99 p.

12. Gaia APO, Leronardo IADG. Habilidades Sociais em Dependentes de Substâncias Psicoativas no Processo de Reinserção Social. Rev Ciênc Amazônia ILES/ ULBRA. [Internet]. 2017. [Acesso 12 ago 2018]; 1(2). Disponível em: http://www.periodicos.ulbra.br/index. php/amazonida/issue/view/192.

13. Feitosa FB. Habilidades sociais e sofrimento psicológico. Arq Bras Psicol. [Internet]. 2013 jun. [Acesso 12 jun 2019]; 65(1): 38-50. Disponível em: http://pepsic.bvsalud.org/scielo.php?script =sci_ arttext\&pid=S1809-52672013000100004\&lng=pt.

14. Caballo VE. Manual de Avaliação e Treinamento das Habilidades Sociais. São Paulo: Santos; 2003. p. 3-6.

15. Bandeira M. Questões conceituais e metodológicas. Campinas. In: Prette A Del, Prette ZAP Del, organizadores. Habilidades Sociais Desenvolvimento e Aprendizagem: Alínea; 2003. p. 207-210.

16. Bandura A. Modificação do Comportamento. Rio de Janeiro: Interamericana; 1979. p. 25-6.

17. Prette A Del, Prette ZAP Del. Psicologia das relações interpessoais: vivências para o trabalho em grupo. Petrópolis: Vozes; 2001. p. 32-6.

18. Bolsoni ATS, Carrara K. Habilidades sociais e análise do comportamento: compatibilidades e dissensões conceitual-metodológicas. Psicol Rev. [Internet]. 2010. [Acesso 16 mar 2018]; 6(2):330-50. Disponível em: doi: 10.5752/P.1678- 9563.2010v16n2p330.

19. Limberger J, Andretta I. Desenvolvimento das Habilidades Sociais na Vida de Mulheres Usuárias de Crack: Estudo de Casos Múltiplos. Temas Psicol. [Internet]. 2017 dez. [Acesso 25 jul 2018]; 25(4):1709-24. Disponível em: http://dx.doi.org/10.9788/tp2017.4-11pt.

20. Prette A Del, Prette ZAP Del. Psicologia das relações interpessoais: vivências para o trabalho em grupo. 11.ed. Petrópolis: Vozes; 2014. p. 63-101.

21. Wagner MF, Oliveira MS. Habilidades sociais e abuso de drogas em adolescentes. Psicol Clín. [Internet]. 2007 dez. [Acesso 25 jul 2018]; 19(2):101-16. Disponível em: http://dx.doi.org/10.1590/S0103-56652007000200008. 22. Wagner MF, Silva JG da, Zanetello LB, Oliveira MS. $\mathrm{O}$ uso da maconha associado ao déficit de habilidades sociais em adolescentes. SMAD, Rev Eletrônica Saúde Mental Álcool e Drog. [Internet]. 2010 ago. [Acesso 25 jul 2018]; 6(2): 255-73. Disponível em: http:// pepsic.bvsalud.org/scielo.php?script=sci_arttext\&pi $\mathrm{d}=$ S1806-69762010000200003.

23. Cunha SM da, Carvalho JCN, Kolling NM, Silva CR da, Kristen $\mathrm{CH}$. Habilidades sociais em alcoolistas: um estudo exploratório. Rev Bras Ter Cogn. [Internet]. 2007 ago. [Acesso 25 jul 2018]; 3(1):31-9. Disponível em: 
http://pepsic.bvsalud.org/scielo.php?script=sci_arttext \&pid $=S 1808-56872007000100004$.

24. Felicissimo FB, Casela ALM, Ronzani TM. Habilidades Sociais e Alcoolismo: uma revisão da literatura. Psicol Estud. [Internet]. 2013 mar. [Acesso 25 jul 2018]; 18(1):137-45. Disponível em: http://www.scielo.br/ scielo. php? pid $=$ S141373722013000100014\&script $=$ sci_abstract\&tlng=t.

25. Limberger J, Andretta I. Habilidades Sociais e Comorbidades Psiquiátricas de Mulheres Usuárias de Crack. Estud Pesqui Psicol. [Internet]. 2017. [Acesso 13 out 2018]; 17(1);103-17. Disponível em: https:// www.e-publicacoes.uerj.br/index.php/revispsi/article/ view/34767/24557.

26. Sa LGC de, Olaz FO, Prette ZAP Del. Propriedades Psicométricas iniciais do Inventário de Habilidades de Enfrentamento Antecipatório para a Abstinência de Álcool e Outras Drogas. Aval Psicol. [Internet]. 2017. [Acesso 25 jul 2018]; 16(2):176-86. Disponível em: http://pepsic.bvsalud.org/scielo.php?script=sci_arttext \&pid=S1677-04712017000200009.

27. Rodrigues VS, Silva JG da, Oliveira MS. Habilidades sociais e tabagismo: uma revisão de literatura. Arq Bras Psicol. [Internet]. 2011. [Acesso 26 jul 2018]; 63(1):31-41. Disponível em: http:// pepsic.bvsalud.org/scielo.php?script=sci_arttext\&pi $d=$ S1809-52672011000100005.

28. Rondina RC, Martins R, Manzato AJ, Terra AP. Habilidades Sociais e Dependência Nicotínica em Universitários Fumantes. PsicOL Saúde Doenças. [Internet]. 2013 mar [Acesso 25 jul 2018]; 14(1):232-44. Disponível em: http://www.scielo.mec.pt/scielo.php?script=sci_arttext\&p id $=$ S1645-00862013000100015.

29. Scnneider JA, Limberger J, Andretta I. Habilidades sociais e drogas: revisão sistemática da produção científica nacional e internacional. Av Psicol Latinoam. [Internet]. 2016 [Acesso 24 jul 2018]; 34(2):339-50. Disponível em: doi: http://dx.doi.org/10.12804/apl34.2.2016.08.

30. Peuker ACWB; Kessler FHP. Transtornos por uso de substâncias. In: Hutz CS, Bandeira DR, Trentini CM, Krug JS, organizadores. Psicodiagnóstico. Porto Alegre: Artmed; 2016. p. 663-8.

31. Zanelatto NA. Terapia cognitivo-comportamental das habilidades sociais e de enfrentamento de situações de risco. In: Zanelatto NA, Laranjeira R. O Tratamento da Dependência Química e as Terapias Cognitivo Comportamentais. Porto Alegre: Artmed; 2013. p. 174-7. 32. Griep RH, Chor D, Faerstein E, Werneck GL, Lopes CS. Validade de constructo de escala de apoio social do Medical Outcomes Study adaptada para o português no Estudo Pró-Saúde. Cad Saúde Pública. [Internet]. 2005 jun. [Acesso 15 maio 2018]; 21(3):703-14. Disponível em: http://www.scielo.br/pdf/\%0D/csp/v21n3/04.pdf.
33. Hutz CS, Zanon C. Revisão da adaptação, validação e normatização da escala de autoestima de Rosenberg: Revision of the adaptation, validation, and normatization of the Roserberg self-esteem scale. Aval Psicol. [Internet]. 2011 abr. [cited Ago 12, 2018]; 10(1):41-9. Available from: http:// pepsic.bvsalud.org/scielo.php?script=sci_arttext\&pi $\mathrm{d}=$ S1677-04712011000100005.

34. Sbicigo JB, Bandeira D, Aglio DDD. Escala de Autoestima de Rosenberg (EAR): validade fatorial e consistência interna. Psico-USF. [Internet]. 2010 set./dez. [Acesso 8 out 2018]. 15(3):395-403. Disponível em: http://www.scielo.br/pdf/pusf/v15n3/v15n3a12.pdf.

35. Prette A Del, Prette ZAP Del. Inventário de Habilidades Sociais. São Paulo: Casa do Psicólogo; 2001. 36. Cardoso LRD, Malberger A. Problemas escolares e o consumo de álcool e outras drogas entre adolescentes. Psicol Esc Educ. [Internet]. 2014 jun. [Acesso 15 out 2018]; 18(1):27-34. Disponível em: http://www.scielo.br/pdf/pee/v18n1/v18n1a03.pdf.

37. Silva ML da, Hatanaka YF, Rondina RC, Silva NR. Avaliação do repertório de habilidades sociais de usuários de substâncias psicoativas em tratamento. Cad Bras Ter Ocup. [Internet] 2018. [Acesso 12 ago 2018]; 26(4):849-58. Disponível em: http://dx.doi. org/10.4322/2526-8910.ctoao1633.

38. Maurina LRC, Cenci CMB, Wagner MF, Martinelli AC, Cerutti PC, Cecconello WW. Habilidades sociais e o abuso de drogas no contexto familiar. Rev Psicol IMED. [Internet] 2012. [Acesso 11 ago 2018]; 4(2):715-22. Disponível em: https://seer.imed.edu.br/index.php/ revistapsico/article/view/241.

39. Prette A Del, Prette ZAP Del. Habilidades sociais e análise do comportamento: Proximidade histórica e atualidades. Perspectivas. [Internet] 2010. [Acesso 18 out 2018]; 1(2):104-15. Disponível em: http:// pepsic.bvsalud.org/scielo.php?script $=$ sci_arttext\&pi $\mathrm{d}=$ S2177-35482010000200004.

40. Schneider JA, Andretta I. Habilidades Sociais de Usuários de Crack em Tratamento nas Comunidades Terapêuticas: Relação com Características Sociodemográficas e de Padrão de Consumo. Rev Col Psicol. [Internet] 2017. [Acesso 30 mar 2018]; 26(1):83-98. Disponível em: http://www.scielo. org.co/pdf/rcps/v26n1/0121-5469-rcps-26-01-00083.pdf. 41. Anini DS, Peixoto EM, Nakano TC. Escala de Apoio Social (MOS-SSS): Proposta de Normatização com Referência nos Itens. Temas Psicol. [Internet] 2018. [Acesso 13 out 2018]; 26(1):387-99. Disponível em: http://www.scielo.br/scielo.php?pid=S2358188320180 00100387\&script=sci_abstract\&tlng=t.

42. Francischetto $V$, Soares AB. Habilidades sociais e estilos de apego em dependentes de substância psicoativa. Psychologica. [Internet] 2014. [Acesso 12 out 2018]; 
57(2). Disponível em: http://impactum-journals.uc.pt/ psychologica/article/view/2312.

43. Sintra CIF, Lopes P, Formiga N. Condutas antissociais e delitivas e habilidades sociais em contexto forense. Psicol Argum. [Internet] 2011. [Acesso 13 out 2018]; 29(66):383-99. Disponível em: https://periodicos.pucpr. br/index.php/psicologiaargumento/article/view/20439.

44. Limberger J, Trinin VR, Hartmann B, Andretta I. Treinamento em habilidades sociais para usuários de drogas: revisão sistemática da literatura. Contextos Clínic. [Internet] 2017 jun. [Acesso 11 ago 2018]; 10(1):99-109. Disponível em: http://dx.doi. org/10.4013/ctc.2017.101.08.

45. Aragão EIS, Campos MR, Portugal FB, Gonçalves DA, Mari JJ, Fortes SLCL. Padrões de Apoio Social na Atenção Primária à Saúde: diferenças entre ter doenças físicas ou transtornos mentais. Cienc Saúde Coletiva. [Internet] 2018 jul. [Acesso 14 out 2018]; 23(7):2339-50. Disponível em: http://dx.doi. org/10.1590/1413-81232018237.21012016.

46. Ribeiro LA., Nappo SA, Sanches ZVDM. Aspectos socioculturais do consumo de crack. In: Ribeiro $M$, Laranjeira $\mathrm{R}$, organizadores. O Tratamento do Usuário de Crack. 2. ed. Porto Alegre: Artmed; 2012. p. 50-5.

47. Laureano BS, Gomes BCQB, Ferreira AS. Avaliação das habilidades sociais com mulheres em tratamento da dependência química. Rev Científica Faesa. [Internet] 2017. [Acesso 7 ago 2018]; 1(13):7-13. Disponível em: <DOI: $10.5008 / 1809.7367 .105>$.

48. Viana AM, Junior GA. Qualidade de vida em idosos praticantes de atividades físicas. Rev Psicol Saúde e Debate. [Internet] 2017 jan. [Acesso 14 out 2018]; 3(1):87-98. Disponível em: http://psicodebate.dpgpsifpm.com.br/ index.php/periodico/article/view/92/76.

49. Horta RL, Schafer JL, Coelho LRM, Rodrigues VS, Oliveira MS, Teixeira VA. Condições associadas a prejuízo de desempenho em habilidades sociais em uma amostra de conveniência de usuários de crack. Cad Saúde Pública. [Internet] 2016. [Acesso 25 jul 2018]; 32(4). Disponível em: http://www.scielo.br/pdf/csp/v32n4/1678-4464csp-32-04-e00010715.pdf.

50. Schneider JA., Andretta I. Habilidades sociais como fatores de risco e proteção entre homens usuários de crack. Quaderns Psicol. [Internet] 2017. [Acesso 21 out 2018]; 19(2): 151-61. Disponível em: https://www. researchgate.net/publication/319437145_Habilidades_ sociais_como_fatores_de_risco_e_protecao_entre_ homens_usuarios_de_crack.

\section{Contribuição dos autores}

Concepção e planejamento do estudo: Adauto Motta Filho, Andresa Aparecida Franceschini de Sousa, Loiane Letícia dos Santos. Obtenção dos dados: Adauto Motta Filho, Andresa Aparecida Franceschini de Sousa. Análise e interpretação dos dados: Adauto Motta Filho, Andresa Aparecida Franceschini de Sousa, Randolfo Dos Santos Junior, Loiane Letícia Dos Santos. Análise estatística: Randolfo Dos Santos Junior, Loiane Letícia Dos Santos. Redação do manuscrito: Adauto Motta Filho, Andresa Aparecida Franceschini de Sousa, Randolfo Dos Santos Junior. Revisão crítica do manuscrito: Adauto Motta Filho, Andresa Aparecida Franceschini de Sousa, Randolfo Dos Santos Junior, Júlio César André, Loiane Letícia Dos Santos.

Todos os autores aprovaram a versão final do texto.

Conflito de interesse: os autores declararam que não há conflito de interesse. 